\title{
Finite Element Analysis of the Loader Boom Based on ABAQUS
}

\author{
Yu-jing He, Ya-dong Tang , Jing-zhao Shi ${ }^{*}$ and $\mathrm{He} \mathrm{Li}$
}

The Agriculture University of Henan, College of Machinery and Electronic Engineering, Zhengzhou, China, 450002

\section{Keywords: Loader; Boom; ABAQUS; Finite Element Analysis; Stress}

\begin{abstract}
Loader Boom Frame is a welding part welded by two long boom plates and cylindroid beam. As condition of loading changes frequently, the beam is the most seriously destructed. Up to now, the analysis of the failure for loader operating device is mainly aimed at the weld fatigue. This paper verified the mechanism for fracture failure of loader boom. Through the force analysis of the dangerous cross section, this paper used ABAQUS for the finite element analysis of ZL50C loader boom and obtained its cloud atlas of stress and region of stress concentration. In accordance of the results, the improvement is put forward to avoid the boom fracture.
\end{abstract}

\section{Introduction}

As a machinery of earthwork construction widely applied in construction projects, such as highway, railway, construction, hydropower, port, and mine, loader is mainly served for shovel-loading soil, gravel, lime, coal, and other bulk materials. the loader boom frame is a welding part welded by two long boom plates and cylindroid beam. As condition of loading changes frequently, the beam is the most seriously destructed. The active arm and beam is welded by circumferential weld, plus the box welding structure of active arm, so welding residual stress and deformation may occur and thus reduce the loader's bearing and operating capacity. The loader boom frame requires bearing some static and load loads when operating, so the welding area of the active arm and beam may have cracks or strength fracture under the cyclic load and load load, causing structural failure at last.

The existing researches on the failure of loader boom are divided into two types: one is on fatigue fracture of the welding joint, and the other one is on boom fracture. Both of them mainly employ the mechanical analysis or virtual simulation. This paper used the ZL50C loader boom frame as the research subject, conducted the mechanical analysis before establishing the finite element model by ABAQUS finite element analysis software, obtained the region of stress concentration and cloud atlas of stress, found out the maximum stress value, and then put forward improvement measures to avoid the boom fracture.

\section{Operating Status of Boom}

The operating mechanism of a loader incorporates 6 parts: scoop cylinder, rocker, boom, bucket, linkage and boom cylinder, which known as the six-bar mechanism [1]. The operating device structure of a loader is shown in Figure 1.The boom rises when a loader operates, and if the boom cylinder remains unchanged, the included angle of bucket and horizontal line would change and thus causes the materials sprinkling.

The typical operating status of a loader can be divided into the following three types:

(1) The loader moves along the horizontal plane, the cylinder of the operating device is closed, the bucket inserts into the material pile, and in this case, the resistance on the bucket by materials is on the main cutting edge horizontally.

(2) After a sufficient depth in materials horizontally, the loader comes to a halt, rotates the bucket or raises the boom, and in this case, the resistance on the bucket by materials is on the cutting edge vertically.

(3) The loader makes uniform motion on the horizontal plane. After a sufficient depth in materials by the bucket horizontally, it inserts into the materials and raises the boom simultaneously, 
and in this case, it is deemed that the resistance on the bucket by materials is on the cutting edge both horizontally and vertically.

The boom of ZL50C loader is made by $16 \mathrm{Mn}$ steel, whose internals are multicrystals. The grains are closely connected, and due to the continuous texture of boom materials, the grains are small and randomly arranged. According to the statistical law of materials, they are both homogeneous and isotropic. Due to the appropriate processing guarantee, when producing, the internals of the boom can be deems to be unstressed in the state of free. Again, during the motion period of the boom, its additional load caused by factors like acceleration and vibration could be ignored. For a boom, its thickness is $60 \mathrm{~mm}$, cylinder length is $960 \mathrm{~mm}$ and cylinder thickness is $20 \mathrm{~mm}$, and the maximum horizontal size is $500 \mathrm{~mm}[2]$.

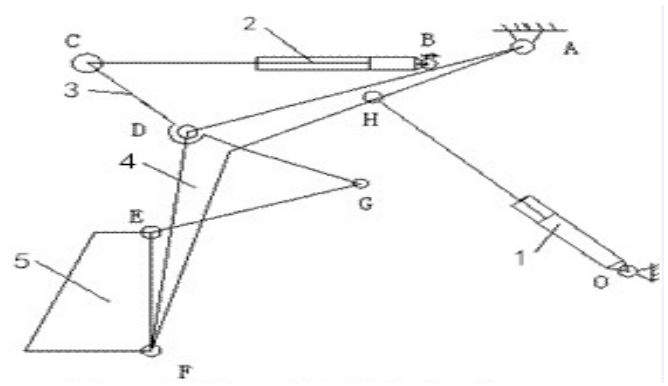

Figure 1. Operating Mechanism of a Loader

1.cylinder 2. Cylinder 3. rocker 4.boom 5.bucket

\section{Load Calculation}

The calculation for resistance of horizontal insert: the maximum inserting resistance on the cutting edge when the operating device is under the condition of single insert is:

$$
F_{f}^{\prime}=F_{d}=G_{\varphi}
$$

In the formula:

G weight when the whole device is zero-load;

$\phi$ coefficient of adhesion of the tire and the ground.

The inserting resistance under the combinational acting condition of inserting and shoveling:

$$
F_{f}=2 / 3 F_{f}^{\prime}=2 / 3 G_{\phi}
$$

The calculation of maximum drawbar pull: because that the maximum drawbar pull is subjected to the power and running speed of the tire transmitted by the loader running system, that is:

$$
F_{f} V_{M} / 1000 \leq P_{a}
$$

In the formula:

$\mathrm{Pa}$ wheel power of the loader

VIM traveling speed when the loader inserts into the materials, generally $0.3 \sim 0.8$.

Therefore, if the above formula satisfies, then $\mathrm{Ff}=\mathrm{Fd}=\mathrm{G} \varphi$; if it fails, then $\mathrm{Ff}=1000 \mathrm{~Pa} / \mathrm{VM}$.

The calculation of vertical shoveling resistance: selects a rated loading capacity, apply the force on the middle part near the prong inside of bucket by following the static equivalent principle. It can be seen from the vertical stability conditions that, the maximum vertical load exerted on the bucket is: 


$$
N=G L_{1} / L
$$

In the formula:

G operating quality of the loader

L1 horizontal distance from the loader center of gravity to the front axle center

L horizontal distance from the front axle center

The calculation of maximum vertical shoveling resistance: the maximum vertical shoveling resistance can be known by the parameter calculation method of loader design:

$$
F_{s b}=2 Q
$$

In the formula:

Q rated loading capacity of the loader

The calculation result of the load: under the condition of uniformly distributed load, the vertical load is $208330 \mathrm{~N}$, while the horizontal load is $223550 \mathrm{~N}$.

\section{FEM Model}

\section{Establishment of FEM model}

Loader Boom Frame is a welding part welded by two long boom plates and cylindroid beam. As condition of loading changes frequently, the beam is the most seriously destructed. The loader boom frame requires bearing some static and load loads when operating, so the welding area of the active arm and beam may have cracks or strength fracture under the cyclic load and load load, causing structural failure at last. This paper established the FEM model in the ABAQUS. The materials and section properties of the boom defined as per Table 1 can be seen in Figure 2.

Table 1. 6Mm Steel Parameters

\begin{tabular}{ccc}
\hline number of shear elastic model $\mathrm{G}$ & poisson ration $\mu$ & yield limit $\delta$ \\
\hline $8.07 \times 104^{4}$ & 0.31 & 361 \\
\hline
\end{tabular}

Figure 2, Boom After Edit Properties

\section{Disposal of Load at the Hinge Joint}

The stress at the hinge joint is not a concentrated force in actual structure, but a force distributed by following the cosine law, as shown in Figure 3. The distributional hypothesis of the cosine load is:

(1) The load is distributed $180^{\circ}$ as per the cosine within the scope of X-Y plane

(2) The distribution force is along the direction of pin hole

(3) The load is uniformly distributed a along the direction of $\mathrm{Z}$. 


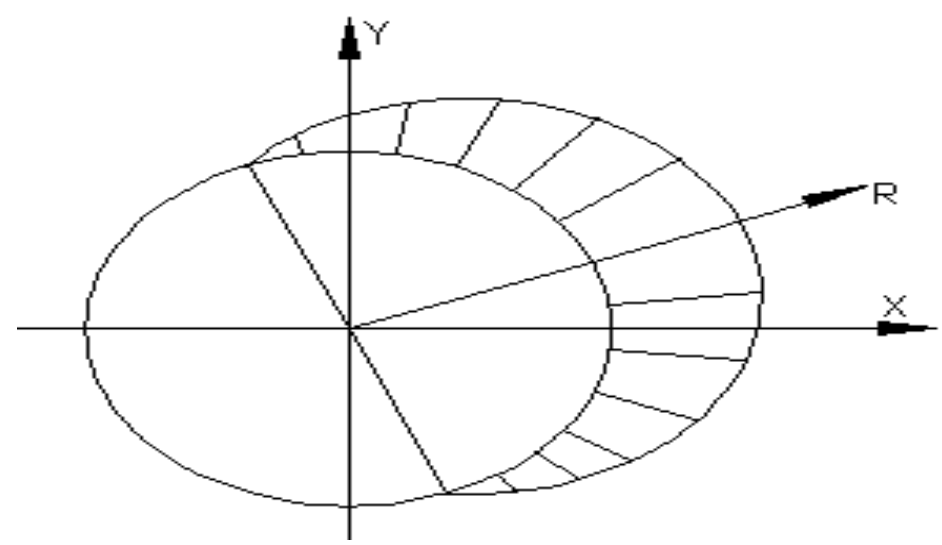

Figure 3, Distribution Diagram of Cosine Load

Assume the distribution function of the load is

$$
F(\theta)=P_{0} \cos (\theta-\alpha)
$$

In the formula:

$\theta$ the included angle between ligature of a point on the pin hole circumference and origin relative to the direction of $\mathrm{x}$ axis, $\theta=0 \sim 180^{\circ} ; \mathrm{P}_{0}$ undetermined coefficient; $a$ included angle of direction of total force and $\mathrm{x}$ axis.

Its total force:

$$
R=\int_{0}^{\pi} F(\theta) \cdot l r \cdot \cos (\theta-\alpha) d \theta=\pi l r P_{0} / 2
$$

Then P0 by reverse calculation can be obtained:

$$
P_{0}=2 R /(\pi l r)
$$

In the formula:

1 length of pin pole; $r$ radius of pin pole; $\mathrm{R}$ total force of cosine load at the pin pole.

The undetermined coefficient P0 can be solved by the formula to obtain load distribution function at thepin pole. In this way, knowing the stress value and direction can apply the surface load of the cosine transform for loads at any hinge joint.

The applied load and defined boundary conditions can be seen in Figure 4. The model regular part is divided into swept grids, while the irregular part is divided into free grids, as shown in Figure 5. According to the statistical data, the model has 19321 units and 9640 nodes after dividing grids.

\section{Results and Post-Processing}

The cloud atlas for stress distribution upon post - processing of boom, as shown in Figure 6, and the region of stress concentration can be thus obtained. It can be seen from the cloud atlas of stress that, the maximum stress point of boom under positive load condition is converged on the upper bracket point-axis and cross linked plane, which is $171 \mathrm{Mpa}$. The maximum stress point of the boom under unbalanced load condition is converged on the section with relatively small boom, upper bracket point-axis and cross linked plane, which is $249 \mathrm{Mpa}$. The $30 \mathrm{MPa}$ stress value on other parts is about 30Mpa, which satisfies the mechanical design requirements and has sufficient margin of strength. 


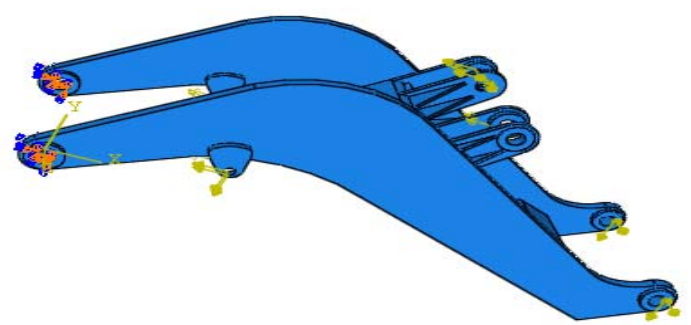

Figure 4, Boom After Load and Defined Boundary Conditions Defined Boundary Conditions

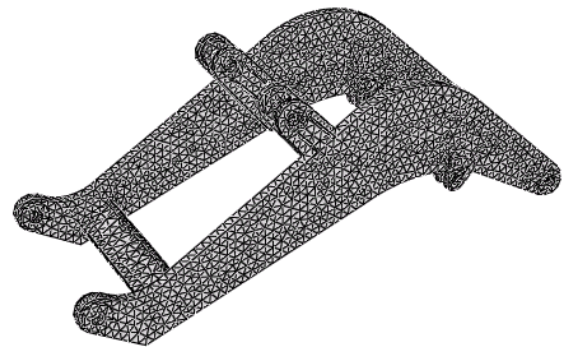

:Figure 5, Boom After Dividing

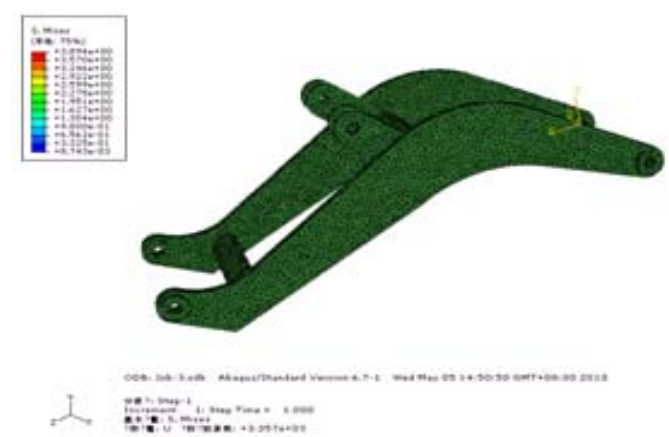

Figure 6, Cloud Atlas of Boom Stress

\section{Conclusions}

It can be seen from the above analysis that, the measures solving the boom fracture should be taken into consideration from the following aspects.

(1) To increase the dangerous section area of the boom and raise the security coefficient of boom dangerous section:

It raises the boom beam by optimization design of boom outline, and thus enhances the rigidity and stability of bucket hinged joint part.

(2) To restore the boom beam to the rigidity of bucket hinged joint part:

The direct means to enhance the beam stability is to change the boom beam position, and translate to the hinged end of bucket. But due to the motion analysis of operating device, it may cause the motion interference, even possibly severe. The change in structural hinge joint of operating device is bound to lead to the reduced universality and scrapped property of parts. Again, it would add the costs of design, manufacture, and after-sale services. As a result, this method is not feasible.

(3) Control from the aspect of processing technology:

One is no allowance of arcing and re-arc strike on the dangerous section of boom plate as well as the sharp flame cutting ripple, and thus to eliminate the stress concentration conditions. The other one is to strictly control the welding variables and welding operation process without any welding deficiencies and thus to ensure the welding quality.

\section{Acknowledgement}

This research was financially supported by Chinese National Soybean Industrial Technology System. 


\section{References}

[1] Zhanhui LI, Yunxin WU, Optimal Design of Loader Reverse-Six-Link Mechanism [J]. Road Machinery \& Construction Mechanization, 2008, (10): 35-43.

[2] Xianguo XU, Yingsi BI, Xin WANG, Control of Combination Welding Deformation for Loader Boom [J]. Journal of Chongqing University, 2004, 27 (3): 13-15.

[3] Hongwen LIU, Mechanics of Materials (Edition IV) [M]. Beijing: Higher Education Press, 2004.

[4] Feng JIANG, Jianfeng LI, Fangyi LI, Yuling WANG, Analyssi for Loader Boom Strength by the Eccentric Load Coefficient Method [J]. China Mechanical Engineering, 2008,19 (3): 272-276.

[5] Nan DUAN, Jiehui LIU, Jiamei WANG, Lijie YANG, Modeling and Simulation Analysis of the Loader Operating Device [J]. Coal Mine Machinery, 2007,28 (5): 70-72.

[6] Jie GONG, Yuxin CUI, Rule Planning for the Loader Excavations [J]. Journal of Mechanical Engineering, 2009, 45 (7): 29-34.

[7] Xijian Zheng, Qi Mang, Zhengyi Xie, Tiejun LI, Simulation Analysis of the Wheel Loader Kinematics and Dynamics Based on ADAMS [J]. Machinery Design and Manufacture, 2009, (2): 206-208.

[8] Haihua LIU, Qingbo SUN, Cause Analysis and Improvement Measures for the Loader Boom Fracture [J]. Agricultural Equipment \& Vehicle Engineering, 2007, (10): 38-39.

[9] Xiaozhao CUI, Finite Element Analysis Strategy Research on Complex Mechanical Structure [D]. Zhejiang: Zhejiang University, 1996.

[10] Zhan LIU, ABAQUS 6.6 Essential Training and Example Analysis [M]. Beijing: Waterpower Press, 2008. 